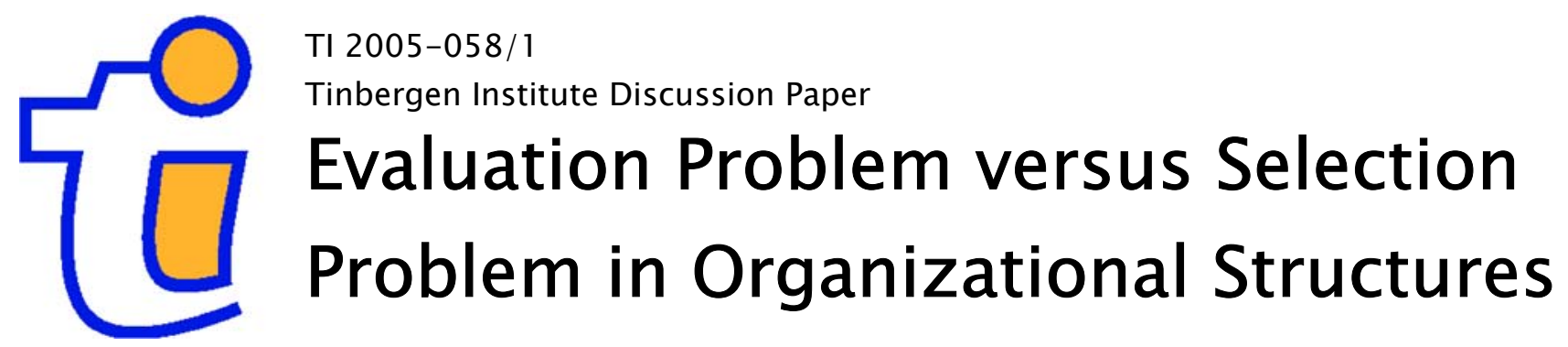

Stefano Ficco *

Vladimir Karamychev

Faculty of Economics, Erasmus University Rotterdam.

* Tinbergen Institute. 


\section{Tinbergen Institute}

The Tinbergen Institute is the institute for economic research of the Erasmus Universiteit Rotterdam, Universiteit van Amsterdam, and Vrije Universiteit Amsterdam.

Tinbergen Institute Amsterdam

Roetersstraat 31

1018 WB Amsterdam

The Netherlands

Tel.: $\quad+31(0) 205513500$

Fax: $\quad+31(0) 205513555$

Tinbergen Institute Rotterdam

Burg. Oudlaan 50

3062 PA Rotterdam

The Netherlands

Tel.: $\quad+31(0) 104088900$

Fax: $\quad+31(0) 104089031$

Please send questions and/or remarks of nonscientific nature to driessen@tinbergen.nl.

Most TI discussion papers can be downloaded at http://www.tinbergen.nl. 


\title{
Evaluation Problem versus Selection Problem in Organizational Structures*
}

\author{
Stefano Ficco \\ (Tinbergen Institute Rotterdam) \\ Vladimir Karamychev \\ (Erasmus University Rotterdam)
}

May 31, 2005

\begin{abstract}
We consider a hierarchical organization with two fully rational agents. The goal of the organization is that of selecting the best alternative out of several available, and agents are heterogenous in the accuracy with which they screen the alternatives. We show that, if internal communications between agents is not possible, the ordering of agents affects the performance of the organization. More specifically, we find that the expected payoff of the organization improves when the more accurate agent screens first. Finally, we note that such optimal ordering makes the hierarchy formally identical to one in which the internal communication flow is perfect.
\end{abstract}

\section{Introduction}

Much economic literature has been devoted to analyze the structure and performance of economic organizations. The main motivation behind this branch of research is the consideration that individuals have limited capability in processing information and, consequently, groups of individuals may be able to perform better than any single individual. An economic organization can be viewed as a decision making network in which each node is its constituent agent. The structure of the organization specifies who gathers information, and who communicates what to whom. The basic feature of an organization is that of aggregating the decisions taken by each one of its members separately. Therefore, it is the specific organizational structure that determines the quality of the final decision undertaken by the organization as a whole.

Since it is natural to assume that agents belonging to the same organization differ in ability (some individuals make, on average, more accurate decisions than

${ }^{*}$ We are indebted to Maarten Janssen for many discussions and helpful suggestions. We also thank seminar participant at Erasmus University for useful comments. 
others) a legitimate question that arises is whether the ordering of heterogenous agents affects the performance of a specific organization. Sah and Stiglitz (1986), and the subsequent literature which follows their approach ${ }^{1}$, provide a negative answer to this question. The common feature of this branch of the literature is that of analyzing the performance of different organizational structures in the context of project evaluation: several projects, whose quality is unobservable, are assumed to come in streams, and the organization evaluates them sequentially in order to distinguish those that are worth implementing from those that are not. However, there exist many examples in which organizations perform a different task. For instance, the goal of many organizations is that of selecting the best out of an arbitrary number of projects (alternatives hereafter) that are simultaneously available. We call this problem, which is different form the one considered by Sah and Siglitz (1986), alternatives selection problem. Consider, for example, the hiring decision of a firm. If the number of vacancies is unlimited then the firm faces an evaluation problem as it must distinguish those workers whose marginal productivity is larger than the wage offered form those with a productivity lower than such a wage. If, on the contrary, only one vacancy is available, then the problem is typically a selection one as the vacancy should be given to the most productive worker.

In the project evaluation framework the terminal payoffs are threefold: the organization gains a profit if it accepts a good alternative, it incurs a loss if it accepts a bad alternative, and gets a payoff of zero if it rejects the alternative. Consequently, the performance of a specific organization is determined by the trade-off between the individuals' errors of not approving good alternatives (Type-I errors) and the errors of approving bad alternatives (Type-II errors). In our alternatives selection framework, on the contrary, the terminal payoffs are twofold: if the selected alternative is good the organizations gets a high payoff (say, one), while, if the selected alternative is bad, the payoff is low (say, zero).

In this paper we focus our attention on a hierarchy because it is the most effective organizational structure to perform the task of selecting the best out of several alternatives. A two-stage selection problem corresponds here to a hierarchical structure where the first agent preselects a subsample of alternatives to be passed to the second stage, and the second agent makes the final selection by choosing one of the preselected alternatives.

A crucial aspect in any organization is the amount of information that can be communicated between agents. It is plausible to think that communication, like decision making, is always imperfect ${ }^{2}$. In order to account for this imperfection we consider two extreme cases, one in which there is perfect communication between agents (denoted by $P C$ ), and one in which there is no communication at all $(N C)$. In our model agents are assumed to be fully rational and heterogenous in the accuracy with which they screen the alternatives.

\footnotetext{
${ }^{1}$ See for example, Hendrikse (1992), Ioannides (1987), Koh (1992a, b, 1993, 1994a, b) and Visser (2000).

${ }^{2}$ For example, communication problems may arise due to the inevitable contamination that occurs in the process of information transmission, or due to a high degree of labour specialization.
} 
We show that, in contrast to the evaluation framework, in the selection framework the performance of an organization is generally affected by the ordering of its agents. We find that the performance of the hierarchy improves when the more accurate agent screens first. Moreover, by letting the best agent act first, the organization overcomes the imperfections due to the lack of internal communication and becomes as efficient as a hierarchy in which the information flow is perfect. Not surprisingly, when communication between agents is perfect, the order in which agents are placed does not matter.

It is important to stress that we evaluate the performance of the organization by its gross expected profit, that is, we assume there is no direct cost involved in processing information. It is indeed the gross expected profit which, in the project implementation literature, turns out to be always unaffected by the ordering of heterogenous agents. If one introduces variable evaluation costs, then the ordering of agents matters also in the standard implementation framework (see for example Koh (1992b)).

The structure of the paper is as follows. In section 2 we present the selection problem, in section 3 we investigate the optimal ordering of agents, in section 4 we provide a link between the evaluation approach introduced by Sah and Stiglitz (1986) and the one ${ }^{3}$ we take here. Section 5 concludes the paper.

\section{The alternatives selection problem.}

There is a population of $N$ alternatives. The quality of each alternative, denoted by $\theta$, can be high or low, $\theta \in\left\{\theta_{H}, \theta_{L}\right\}$. A high quality alternative yields utility of 1 , while a low quality alternative yields utility of 0 . The share of high-type alternatives in the population is denoted by $\alpha$. The organization we consider is a hierarchy with two agents denoted by 1 and 2 . Agent 1 screens all $N$ alternatives and decides which ones to pass to agent 2 . Agent 2 screens the subsample preselected by agent 1 and eventually selects one alternative. The expected quality of the alternative selected by agent 2 determines the payoff of the organization. Each agent in the model is interpreted to be Bayesian, with each of them receiving an imperfect binary signal (high or low) about the quality of each alternative. A signal observed in stage $t$ is denoted by $x_{t} \in\left\{h_{t}, l_{t}\right\}$, with $t=1,2$ and we assume that

$$
\operatorname{Pr}\left(h_{t} \mid \theta_{H}\right)=\operatorname{Pr}\left(l_{t} \mid \theta_{L}\right)=q_{t} \in(1 / 2,1)
$$

Assumption (1) implies that signals have the monotone likelihood ratio property (MLRP), where $q_{t}$ is the revealing probability of a signal observed in stage $t$. Notice that $q_{t}$ denotes also the screening accuracy of agent $t$. Finally, we assume that all signals in stage 1 and 2 are statistically independent

$$
\operatorname{Pr}\left(x_{1}, x_{2} \mid \theta_{i}\right)=\operatorname{Pr}\left(x_{1} \mid \theta_{i}\right) \operatorname{Pr}\left(x_{2} \mid \theta_{i}\right), i=H, L
$$

\footnotetext{
${ }^{3}$ The alternatives selection framework is adopted also in Ficco and Karamychev (2004). There the authors investigate the nature of the optimal strategy profile under different information-processing limitations.
} 
We model the $P C$ case by assuming that agent 2 observes not only the signal outcomes produced in stage 2 but also those produced in stage 1 . By doing so, agent 2 makes his choice based on signals produced in both stages and use the pair $\left(x_{1}, x_{2}\right)$ to determine the overall likelihood value for each alternative ${ }^{4}$. On the contrary we assume that, in $N C$ case, agent 2 makes his choice based only on signals $x_{2}{ }^{5}$.

The strategies of the two agents are as follows. Agent 1, having observed the signal outcomes of stage 1 , passes a number of $h_{1}$-alternatives and a number of $l_{1}$-alternartives to stage 2 . Agent 2 observes the signal outcomes of stage 2 (and of stage 1 in case of $P C$ ) and eventually selects the final alternative. The selection rule of the hierarchy, is therefore defined by the strategy profile $S=\left\{s_{1}, s_{2}\right\}$, where $s_{1}$ and $s_{2}$ are the strategies sequentially implemented by each one of the two agents ${ }^{6}$.

The exogenous parameters $\alpha, q_{1}$ and $q_{2}$ induce a distribution over the $(N \times 2)$ matrix of binary signal outcomes, $\mathbf{X} \equiv\left[x_{j t}\right] \equiv\left[\mathbf{x}_{1}, \mathbf{x}_{2}\right]$, where $j=1, . ., N$ is an arbitrary alternative, $t=1,2$ is the signalling stage, and $\mathbf{x}_{1}$ and $\mathbf{x}_{2}$ are the column vectors of signal realizations in stage 1 and stage 2 respectively. The specific matrix realization $\mathbf{X}$ and the strategy profile $S$, determine the probability that each alternative $j$ is eventually selected. When alternative $j$ is selected the terminal payoff is $\operatorname{Pr}\left(\theta_{j}=\theta_{H} \mid x_{j 1}, x_{j 2}\right)$, therefore, the expected payoff of the organization is

$$
u=\sum_{\mathbf{X} \in \mathbb{X}} \operatorname{Pr}(\mathbf{X}) \cdot \sum_{j=1}^{N} \operatorname{Pr}(j \text { is selected } \mid \mathbf{X}) \cdot \operatorname{Pr}\left(\theta_{j}=\theta_{H} \mid x_{j 1}, x_{j 2}\right)
$$

where $\mathbb{X}$ is the set of possible matrix realizations. The last probability in (3) is a standard bayesian update of the prior. The probability in the middle of (3) is, on the other hand, a function of the strategy profile $S$ and the information environment. Then, in the $P C$ case

$$
\begin{aligned}
\operatorname{Pr}(j \text { is selected } \mid \mathbf{X})= & \operatorname{Pr}\left(j \text { is selected in stage } 1 \mid \mathbf{x}_{1}\right) \times \\
& \times \operatorname{Pr}\left(j \text { is selected in stage } 2 \mid \mathbf{x}_{1}, \mathbf{x}_{2}\right)
\end{aligned}
$$

while in the $N C$ case

$$
\begin{aligned}
\operatorname{Pr}(j \text { is selected } \mid \mathbf{X})= & \operatorname{Pr}\left(j \text { is selected in stage } 1 \mid \mathbf{x}_{1}\right) \times \\
& \times \operatorname{Pr}\left(j \text { is selected in stage } 2 \mid \mathbf{x}_{2}\right)
\end{aligned}
$$

Finally, we assume that agents are fully rational and that the distribution of types and the structure of the organization are common knowledge.

\footnotetext{
${ }^{4}$ One can think of agent 1 and agent 2 as two engineers each one evaluating a different feature of several projects. Given the common background, agent 2 can read and understand the report made by agent 1 about each preselected project.

${ }^{5}$ Think of agent 1 is an engineer and agent 2 as a salesman. The specialization of labour makes it impossible for the salesman to understand the technical reports written by the engineer.

${ }^{6}$ For a complete analysis on the nature of optimal selection strategies in multi-stage selection games, see Ficco and Karamychev (2004).
} 


\section{The optimal ordering of agents.}

In this section we investigate whether the ordering of heterogenous agents affects the performance of the organization. We model heterogeneity by assuming that one agent has a high screening accuracy, $q^{H}$ while the other has a low screening accuracy, $q^{L}<q^{H}$. We denote by $u\left(q^{H}, q^{L}\right)\left(u\left(q^{L}, q^{H}\right)\right)$ the expected payoff of the hierarchy when the agent with better accuracy acts first (second).

We start from the benchmark $P C$ case and, not surprisingly, we find that the performance of the hierarchy is not affected by the ordering of its agents.

Proposition $1 u^{P C}\left(q^{H}, q^{L}\right)=u^{P C}\left(q^{L}, q^{H}\right)$

Proof. The perfect communication environment and the rationality of agents ensure that passing all alternatives to the second stage is always optimal, and that the optimal selection rule $S$ can be represented by a correct preference relation over all possible signal pairs. If $q_{1}=q^{H}>q^{L}=q_{2}$ the preference relation is

$$
\left(h_{1}, h_{2}\right) \succ\left(h_{1}, l_{2}\right) \succ\left(l_{1}, h_{2}\right) \succ\left(l_{1}, l_{2}\right)
$$

If, on the contrary $q_{1}=q^{L}<q^{H}=q_{2}$, the preference relation is

$$
\left(h_{1}, h_{2}\right) \succ\left(l_{1}, h_{2}\right) \succ\left(h_{1}, l_{2}\right) \succ\left(l_{1}, l_{2}\right)
$$

Such preferences ensure that the hierarchy ranks correctly the rows (alternatives) of the signal realizations' matrix, and eventually selects the one with the higher overall likelihood of being of high type. Since $\operatorname{Pr}(\mathbf{X})=\Pi_{j=1}^{N} \operatorname{Pr}\left(x_{1 j}, x_{2 j}\right)$, it follows form Bayes' rule that the probability of observing the matrix $\left[\mathbf{x}_{1}, \mathbf{x}_{2}\right]$, conditional on $q_{1}=q^{H}$ and $q_{2}=q^{L}$, equals the probability of observing the matrix $\left[\mathbf{x}_{2}, \mathbf{x}_{1}\right]$, conditional on $q_{1}=q^{L}$ and $q_{2}=q^{H}$. That is, given an arbitrary matix, inverting the order of $q^{H}$ and $q^{L}$ induces an identical probability over the matrix in which the order of the columns is also inverted. Such symmetry, together with the symmetry of preferences $P 1$ and $P 2$, and the fact that the terminal payoff $\operatorname{Pr}\left(\theta_{H} \mid x_{1}, x_{2}\right)$ does not depend on the order in which $q^{H}$ and $q^{L}$ are placed, implies that, indeed, $u^{P C}\left(q^{H}, q^{L}\right)=u^{P C}\left(q^{L}, q^{H}\right)$.

We consider now a hierarchy in which communication between agents is not possible. We find that, here, the ordering of agents affects the performance of the organization: by having the best screener in the first place the hierarchy achieves a higher payoff. Moreover, by letting the best agent act first, the hierarchy overcomes the imperfections due to the lack of internal communication and turns out to be as efficient as a hierarchy in which the information flow is perfect.

Proposition $2 u^{N C}\left(q^{L}, q^{H}\right)<u^{N C}\left(q^{H}, q^{L}\right)=u^{P C}\left(q^{H}, q^{L}\right)$

Proof. If $q_{1}=q^{H}>q^{L}=q_{2}$ the preference relation over all possible signal pairs is

$$
\left(h_{1}, h_{2}\right) \succ\left(h_{1}, l_{2}\right) \succ\left(l_{1}, h_{2}\right) \succ\left(l_{1}, l_{2}\right)
$$


It is clear from $(P 1)$ that selecting only $h_{1}$-alternatives is an optimal strategy for agent 1 . The reason is that, independently of the signal realizations of stage $2, l_{1^{-}}$ alternatives will never produce an overall likelihood value larger than that of $h_{1-}$ alternatives. Since it is optimal for agent 2 to select a $h_{2}$-alternative, the optimal strategy profile ensures indeed that the alternative eventually selected is the one with the higher likelihood value. This proves $u^{N C}\left(q^{H}, q^{L}\right)=u^{P C}\left(q^{H}, q^{L}\right)$. If, on the contrary, $q_{1}=q^{L}<q^{H}=q_{2}$, the preference relation is

$$
\left(h_{1}, h_{2}\right) \succ\left(l_{1}, h_{2}\right) \succ\left(h_{1}, l_{2}\right) \succ\left(l_{1}, l_{2}\right)
$$

Assume first that agent 1 passes only $h_{1}$-alternatives. With positive probability all preselected alternatives produce signals $l_{2}$ and at least one $l_{1}$-alternative produces a signal $h_{2}$. When this event occurs the payoff-dominant pair $\left(l_{1}, h_{2}\right)$ cannot be selected because it has been previously discarded by agent 1 . Assume now that some (possibly all) $l_{1}$-alternatives are passed in addition to $h_{1^{-}}$ alternatives . There exists a positive probability that all preselected alternatives generate identical signals in stage 2 making it impossible for agent 2 to select the payoff-dominant alternative with probability 1 . Thus, for any strategy adopted by agent 1 , there is always a chance that the alternative with the higher overall likelihood value is not selected. This proves $u^{N C}\left(q^{L}, q^{H}\right)<u^{N C}\left(q^{H}, q^{L}\right)$.

The intuition behind proposition 2 is clear. If the more accurate screener acts first, he does not need to pass to the second stage alternatives that produced a low signal because, independently of the signal realizations of stage 2 , they will never produce a likelihood value larger than that of alternatives that produced a high signal in stage 1 . Therefore agent 2 knows that he always receives a subsample of projects with identical $x_{1}$-signal outcomes. Consequently, he does not need any explicit information about the signal outcomes of stage 1 in order to make the right choice: by selecting an alternative that produced a high signal in stage 2, agent 2 automatically selects the best (in expected terms) alternative.

Such argument can be generalized to an arbitrary number of stages, though, a more stringent requirement over the relative differences in agents' accuracy is needed. If there are $T$ selection stages, ordering the agents in terms of decreasing accuracy $q_{1} \ldots<q_{t}<\ldots<q_{T}$, solves the problems due to imperfect communication only if, at any stage $t$, it is optimal for agent $t$ to pass only $h_{t}$-alternatives. Such strategy is indeed optimal provided that $q_{t}$ is such that $\operatorname{Pr}\left(\theta_{H} \mid h_{t}, l_{t+1}, . ., l_{T}\right) \geq \operatorname{Pr}\left(\theta_{H} \mid l_{t}, h_{t+1}, . ., h_{T}\right)$, for any $t=1, . ., T$.

\section{Evaluation versus selection.}

We show here how the traditional project evaluation problem can be restated in terms of our selection model. Consider the problem of deciding whether or not to implement a project of unknown quality, $\theta$. An organization facing such a problem is actually undecided between two alternatives: alternative 1 stands for "implement the project" while alternative 2 stands for "do not implement the project". Therefore an evaluation problem can be restated as a selection problem 
between two alternatives, provided we impose the following restrictions on signal outcomes

$$
\begin{gathered}
\operatorname{Pr}\left(h_{1 t} \mid \theta_{H}\right)=\operatorname{Pr}\left(l_{1 t} \mid \theta_{L}\right)=q_{t} \in(1 / 2,1), t=1,2 \\
\operatorname{Pr}\left(h_{1 t} \mid l_{2 t}\right)=\operatorname{Pr}\left(h_{1 t} \mid l_{2 t}\right)=1, t=1,2
\end{gathered}
$$

Condition (4) means that, it the project is good (bad), then each screening stage is more likely to suggest in favor of implementing (not implementing) the project. Moreover, (5) means that signal outcomes across alternatives are perfectly correlated: if the report of a screener is in favor of implementing the project it is necessarily against not implementing it, and vice versa. Notice that this new formulation is a more restricted ${ }^{7}$ model than the one presented in section 2 as, here, we do not allow for the possibility that two alternatives generate identical signals. This also means that $1-q_{t}$ is the probability that screener $t$ is inclined to accept (reject) a bad (good) project and the standard analysis of the trade off between Type-I and Type-II errors can be applied.

\section{Conclusion.}

In this section we stress in more details the differences between the assumptions we use in the paper and those present in the existing literature. In most of the literature (see for example, Hendrikse (1992), Ioannides (1987), Koh (1992a, b, 1993, 1994a, b) Sah and Stiglitz (1986)) it is assumed that agents are not rational. They are characterized by a pair of probabilities with which they accept good and bad alternatives. These probabilities are determined exogenously and do not reflect the organizational structure, nor the positions in which agents are placed. Visser (2000), instead, comes close to our approach as he also assumes fully rational heterogeneous individuals that cannot communicate the information they posses. However, in Visser (2000) the ordering of agents does not affect the expected payoff of the organization (proposition 3 and proposition 5 in his paper). The intuition behind this result is the symmetry of the organizational decision problem and of the knowledge agents have about the structure and each other's rationality. The novel selection framework we use here is responsible for the different results we obtain. Individual rationality itself is not sufficient to preserve the symmetry of the organizational decision problem. For this to be the case communication must be perfect, otherwise such symmetry fails to hold. Our analysis also provides a solution to this problem as we show that the lack of communication can be overcome by an appropriate ordering of the agents.

Finally, one may argue that, in real life, there exist also examples in which the more accurate screening stages are placed at higher hierarchical levels. The finding of our paper seems to address that such practice is not optimal. However, it is important to stress that we didn't account for the direct costs usually involved in any information-processing phase. As it is natural to assume that

\footnotetext{
${ }^{7}$ It is more restricted because we impose perfect correlation between signals of two different alternatives: if one is high the other is necessarily low, and vice versa.
} 
the cost of processing information increases with the accuracy with which information is processed, it might be the case that having higher accuracy at higher stages is optimal for the simple reason that, at later stages, less information is processed. Whenever such ordering is in place, the contribution of our paper is that of identifying the indirect cost that might emerge due to a lack of internal communication.

\section{References}

[1] Ficco, S., and V. Karamychev (2004). Information Overload in Multi-stage Selection Procedures. Tinbergen Institute Discussion Paper.

[2] Hendrikse, G.W.J., (1992). Competition between Architectures. Annales d'Economie et de Statistique, 25/26, 39-50.

[3] Ioannides, Y.M., (1987). On the Architecture of Complex Organizations. Economics Letters, 25, 201-206.

[4] Koh, W.T.H., (1992a). Human Fallibility and Sequential Decision Making. Hierarchy versus Polyarchy. Journal of Economic Behavior and Organization, 18, 317-345.

[5] Koh, W.T.H., (1992b). Variable Evaluation Costs and the Design of Fallible Hierarchies and Polyarchies. Economics Letters, 38, 313-318.

[6] Koh, W.T.H., (1993). First-Mover Advantage and Organizational Structure. Economics Letters, 43,47-52.

[7] Koh, W.T.H., (1994a). Making Decisions in Commitees. A Human Fallibility Approach. Journal of Economic Behavior and Organization, 23, 195-214.

[8] Koh, W.T.H., (1994b). Fallibility and Sequential Decision-Making. Journal of Institutional and Theoretical Economics, 150, 362-374.

[9] Sah, R. K., and J. E. Stiglitz (1986). The Architecture of Economic Systems: Hierarchies and Polyarchies. American Economic Review, 76, 716-727.

[10] Visser, B.,(2000). Organizational Communication Structure and Performance, Journal of Economic Behavior and Organization, 42, 231-252. 